\title{
The Finnish processing pipeline during nuclear or radiological emergency preparedness and response
}

\author{
M. AMMANN, T. PELTONEN, J. LAHTINEN, K. VESTERBACKA
}

\begin{abstract}
During nuclear or radiological emergency preparedness and response a processing pipeline is put in place at the Finnish Radiation and Nuclear Safety Authority to produce assessment reports of the current situation, its likely evolution with time, and recommendations on protective actions. Within the processing pipeline raw data is more and more refined towards these end products as experts, advisors, and decision makers collect, analyze, portray, annotate, filter, arrange and rearrange, share, approve or dismiss, and publish content. Emergency procedures and guidelines assist and guide them through the process, as do information and communication technologies. The inner working of the processing pipeline is in constant flux as information and communication technologies develop and allow better facilitation of the process, and as social and political demands change. The present article describes the overall working of the pipeline, and how it has recently been improved by the KETALE collaborative software. The focus then shifts to the new ICRP recommendations and how the new national emergency guidelines are planned to be implemented within the KETALE software. The KETALE software has greatly increased the throughput of our emergency processing pipeline and improved product quality.
\end{abstract}

Keywords: Processing pipeline/content management/collaboration/emergency

\section{Introduction}

The Radiation and Nuclear Safety Authority (STUK) plays an important role in Finland in nuclear or radiological preparedness and during response. To be prepared it has to develop and train an efficient organizational structure, operate an emergency centre, publish guides, prepare emergency plans and procedures, and keep contact with stakeholders and decision makers. In response situations it activates its emergency organization and acts as a competent authority and national

STUK, Radiation and Nuclear Safety Authority, Laippatie 4, 00881 Helsinki, Finland. 
warning point. The tasks of STUK are to give expert advice, inform the public, arrange press briefings about the state of affairs and likely developments, and recommend protective actions. In order to do so it has to process available measurement data, run model simulations and analyze their results, collect and evaluate information from the plant and from other sources, collect background information (geographic and demographic information, statistical data, and other facts), and - to a certain extent - make value judgments.

Emergency response is a collective effort and relies, amongst other things, on effective information flows and communication. At STUK we have been developing in cooperation with the Finnish Meteorological Institute (FMI) a content management system that makes it easier to exchange information and for all users to be kept up-to-date with the development of the situation (Ammann et al., 2008, 2010, 2011a, 2011b; Lahtinen et al., 2008; Peltonen and Ammann, 2011). The application bridges the spatial remoteness of expertise and provides a consistent user interface to tools that could be used before only with special training. It supports the process from source term input, over dispersion and dose assessments, up to strategy planning and the dissemination of recommendations on protective actions. This application is described in more detail in the following section.

The process is constantly reformed by societal changes and by advances in information and communication technologies (ICT). Societal changes, for example, have precipitated in the form of new international and national recommendations (ICRP, 2007, 2009a, 2009b; STUK, 2011a, 2011b), which have to be accommodated at certain stages within the process. How this is planned to be done at STUK is described in the section thereafter. New technological solutions allow, as will be seen, novel and streamlined implementations of the process. For example, the use of modern IC technologies is a precondition to integrate spatially remote expertise (incl. hardware and software) into one coordinated process.

\section{Processing pipeline and document management}

The emergency management process that is activated at STUK during nuclear or radiological preparedness and response situations can be viewed as a processing pipeline, within which raw data is more and more refined towards the end products of assessment reports, recommendations, press releases, and the like. A pipeline is a series of processing stages through which data flow, just as water flows through the sections of a water pipe. In a pipeline, a complex task is performed by processing data through several stages in an appropriate sequence. A pipeline is thus a set of data processing stages connected in series, so that the output of one stage is the input of the next one.

The information that flows in the pipeline can be understood as a stream of documents. Here we use the term document in a broad sense: documents are everything that is processed in the pipeline. Mostly they are text documents (reports, assessments, notifications) but they can also be the output of model calculations or 
measurement data. During past preparedness and response events we encountered at least the following kind of documents:

- Log messages, which keep track of who was doing what and when;

- Notifications with the purpose of alerting, warning, informing the emergency organization of certain things. For example, the fact that plant emergency has been declared might be communicated to the whole emergency organization; or FMI might want to inform STUK that a particular numerical weather prediction model is favorable in the prevailing situation;

- Site, reactor, inventory, and release documents: these documents describe, respectively, the site (geographic coordinates), the reactor (burn-up, thermal power, current status), the inventory (nuclide activity of the core), and release (the time, amount, and composition of released material);

- Model requests and model results: request documents contain the input parameters for requested models, and results contain the result data and accompanying meta-data;

- Measurement document: this is a document that lists measurement results (sample, time, location, value, unit);

- Recommendation and strategy: a strategy document contains a table with state, action, administrative unit - columns (see Fig. 1); a recommendation document has recommendation, rational, and instruction sections.

- Report: a report may consist of several sections; each section has a header and variable text content; images and tables can be included;

- Ticket: this is a document that is used to assign certain tasks to certain addressees.

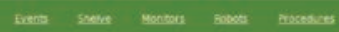

\section{Ketale}

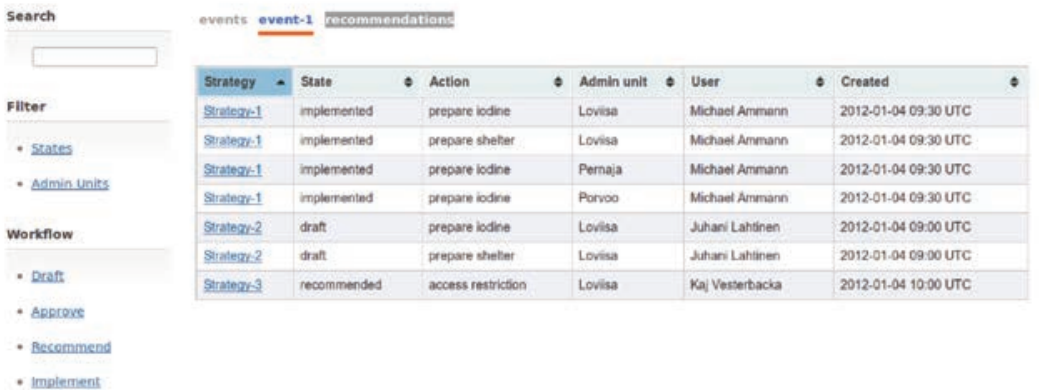


Common to all processed documents are certain elements:

- Documents need to be identifiable and locatable within the stream.

- Documents are interrelated, and may be derivatives from each other. Documents often aggregate information from many sources. For example, recommendations are often based on dispersion calculations, which in turn are based on release assessments and weather model selections.

- Documents have a life cycle within the process. For example, a strategy is first drafted, then approved, recommended and, depending on circumstances and decisions made outside the boundaries of our process, implemented or not.

- Documents have common meta-data elements (ID, title, state, tags, type, author, time-stamp), but the content is variable and not always foreseeable.

The stream is facilitated by the KETALE application, which is a book-keeping system for event related documents. All documents (both incoming and processed) are registered in the system with accompanying meta-data and can be listed along a time-line. A new or updated document appears at the top of the time-line together with the name of the author, the type of document, and the state it is in. This book-keeping system has many similarities with a content management system. It provides the following features:

- Finding documents is made easy by a tagging and classification system. The stream of documents can be filtered (by state, type, tag, author) and documents can be searched in full for the appearance of arbitrary strings.

- Documents are interlinked as acyclic graphs (i.e. in a tree structure).

- All sort of documents can be stored in the schema-less database that is used.

- The document history is preserved.

- Documents take part in workflows.

- The visibility and accessibility of documents is governed by fine grained access control.

The KETALE application can react appropriately to the various types of documents due to semantic markup of certain content elements. The content of documents containing coordinates (for example site or measurement documents) can be displayed on top of geographic maps; the results of dispersion and dose calculations can be interactively portrayed and the portrayals added to reports; strategies can be interactively planned on top of choropleth maps of some relevant quantity (e.g. annual dose, or maximal dose-rate).

Within the pipeline metaphor KETALE implements the (virtual) stream of documents. The pipeline is more than that: it consists of the emergency organization and attributable infrastructure, in short everything that is part of the process. But what is crucial to the emergency management process is its scalability and adaptability to partly unforeseeable future events. Both of these requirements are 
made possible by emergency procedures that are not a rigid set of rules, but give room to assessment, evaluation and value judgments. Procedures allow modifications to the process without necessarily requiring organizational or infrastructural changes.

\section{Strategies and recommendations}

Our emergency procedures are under constant revision, especially now as STUK is in the process of updating its emergency guides (STUK, 2012a, 2012b; Mustonen, 2012) in order to take account of the 2007 Recommendations of the ICRP (ICRP, 2007). The 2007 Recommendation brought about a few notable changes that have an impact on the emergency process:

- Protective actions should be considered not in isolation, but together as strategies.

- The optimization process should be constrained by an upper annual dose limit (termed reference level in the case of emergency and existing exposure situations).

- The relevant quantity to be compared to the reference level is the residual dose (annual projected dose).

STUK's emergency guides set $20 \mathrm{mSv}$ as reference level. The aim is not let this annual dose constraint to be exceeded by implementing appropriate protective actions. Operational intervention levels are defined for selective actions. For example, sheltering indoors is advised when the dose is expected to exceed $10 \mathrm{mSv}$ after two days; or iodine prophylaxis is advised when the dose to the thyroid is expected to exceed $100 \mathrm{mGy}$ in adults, and $10 \mathrm{mGy}$ in children. Instead of these dose values the ambient dose-rate is mostly used, however, as a measurable proxy (STUK, 2012a).

The 2007 Recommendation reinforces the need to prepare emergency plans in advance. Detailed emergency plans are prepared in the precautionary action zone (up to about $5 \mathrm{~km}$ from the site) and urgent protective action zone (roughly between 5 and $20 \mathrm{~km}$ from the site), and are triggered by the declaration of general emergency, and by plant status and wind measurements, respectively. However, farther away it is seldom possible to prepare a detailed emergency plan ahead of time. This can only be done just-in-time with actual numerical weather data and postulated release scenarios (typically by use of dispersion models). Postulated release scenarios are needed because in the pre-release phase the release characteristics (begin, duration, amount, composition) are typically not known with any usable accuracy. In such cases, one might postulate a worst case scenario and use it for warning and preparation purposes, and a few other (probably more realistic) scenarios to prepare action plans.

There remains the problem of how to effectively define strategies and draft recommendations. Here is how this is planned to be supported by the KETALE 
application. In a first approximation, we offer a choropleth map of the relevant quantity to be compared to the operational intervention level. On top of that are selectable shapes of administrative units. By clicking on them protective actions can be selected. In a second approximation, we let software calculate maps of strategies, and allow users to modify them. In a final approximation, we will assess the residual doses of each strategy and check conformance with the dose constraint.

A strategy is defined by a table with state, action, and administrative unit - columns. A strategy document goes - according to its workflow definition - through several states: from draft, over approved and recommended to implemented or rejected and finally to ceased. Actions are phrases like prepare iodine tablets or implement shelter. In order to follow up the development and state of strategies, a table like the one shown in Figure 1 is available. This table can be filtered and sorted by columns.

Given an approved strategy definition, it is scheduled to be recommended as the next step. For this a recommendation is issued that explains the strategy in simple language and provides a rational, and possibly instructions. Well deliberated templates are available for all state, action - combinations in Finnish, Swedish, and English.

\section{Conclusions}

The KETALE software has greatly increased the throughput of our emergency processing pipeline and improved product quality. In doing so it preserves a complete audit trail of all digested documents. Data and information exchange is streamlined, transparent, traceable, and routinely tested. Reports are produced much faster than before, they are standardized and better deliberated, and data portrayals are tailored to the needs of the recipients.

The pipeline metaphor is useful because it helps to focus on the throughput of the pipeline, i.e. the overall performance of the process. Though pipelining does not per se decrease the time for a single document to be processed, it increases the throughput of the system when processing a stream of documents. The pipelined process outputs finished items at a rate determined by its slowest part. The metaphor also helps to see collaboration in a new light. Collaboration is achieved by taking a document from the stream for processing, and returning it (or a derivative document) to the stream thereafter. End products of the process aggregate thus contributions from the various processing stages.

To prepare several action plans just-in-time requires an effective emergency process (i.e. a high throughput pipeline). Our KETALE software helped streamlining our emergency process. In doing so it follows closely our emergency management process (e.g. users, roles, workflow, document types). One of the main design criteria was usability. No installation is required by the users as the system can be accessed by any modern web browser. The system provides a consistent user interface, and it is constantly tested. Although still in development, we have received very positive feedback during past exercises. 
Acknowledgement. There have been many more persons involved in the KETALE project over the years than could have been accommodated in the list of authors. We thank the project members H. Lemmelä from STUK, and M. Seppänen, T. Summanen, A. Sarkanen, M. Rantamäki, J. Vira, J. Korhonen, M. Aalto from FMI. We thank the members of the steering group H. Aaltonen, R. Mustonen from STUK, and J. Hyrkkänen and T. Riihisaari from FMI.

\section{Disclaimer}

The views presented here represent the views of the authors and do not necessarily represent the official view of STUK.

\section{REFERENCES}

Ammann M., Peltonen T., Ilander T., Kuukankorpi S., Lahtinen J., Lemmelä H., Rantamäki M., Salonen T., Sarkanen A., Seppänen M., Siljamo P., Summanen T., Vesterbacka K. (2008) Design of the KETALE web application to improve collaboratorive emergency management. In: Proceedings of the NSFS XV conference, May 26-30, 2008, Ålesund, Strålevern Rapport 2008:13 pp. 162-167. Østerås: Norwegian Radiation Protection Authority.

Ammann M., Peltonen T., Lahtinen J., Vesterbacka K., Summanen T., Seppänen M., Siljamo P., Sarkanen A., Rantamäki M. (2010) KETALE Web application to improve collaborative emergency management. In: 7th International Conference on Information Systems for Crisis Response and Management. Defining Crisis Management 3.0, May 2010, Seattle.

Ammann M., Peltonen T., Lahtinen J. (2011a) Outlines of a content management system for nuclear emergency preparedness and response. In: Current challenges in radiation protection, August 22-25, 2011, Reykjavik.

Ammann M., Peltonen T., Lahtinen J., Vesterbacka K., Rantamäki M., Sarkanen A., Seppänen M., Siljamo P., Summanen T. (2011b) Collaborative software for the nuclear emergency management. In: Third European IRPA Congress, June 14-18, 2010, Helsinki.

ICRP Publication 103 (2007) The 2007 Recommendations of the International Commission on Radiological Protection, Ann. ICRP 37 (2-4).

ICRP Publication 109 (2009a) Application of the Commission's Recommendations for the Protection of People in Emergency Exposure Situations, Ann. ICRP 39 (1).

ICRP Publication 111 (2009b) Application of the Commission's Recommendations to the Protection of People Living in Long-term Contaminated Areas after a Nuclear Accident or a Radiation Emergency, Ann. ICRP 39 (3). 
Lahtinen J., Ammann M., Ilander T., Kuukankorpi S., Lemmelä H., Peltonen T., Rantamäki M., Salonen T., Sarkanen A., Seppänen M., Siljamo P., Summanen T., Vesterbacka K. (2008) KETALE - A centralised data system for the management of dispersion and dose calculation results. In: International Conference on Radioecology and Environmental Radioactivity, June 15-20, 2008, Bergen, Østerås: Norwegian Radiation Protection Authority (Strand P., Brown J, Jølle T. Eds) pp. 142-145.

Mustonen R. (2012) Planned reference and intervention levels in Finland. Submitted to Radiationprotection.

Peltonen T., Ammann M. (2011) Georeferenced data sharing during radiological accidents. In: Current challenges in radiation protection, August 22-25, 2011, Reykjavik.

STUK VAL1 (2011a) Protective measures in the early phase of a nuclear or radiological emergency. STUK GUIDE VAL1, unpublished draft.

STUK VAL2 (2011b) Protective measures in the post-release phase of a nuclear or radiological emergency. STUK GUIDE VAL2, unpublished draft. 